\title{
Real Time Detection and Identification of Human Emotions through Live Streaming
}

\author{
Keerthana B.Chigateri
}

\begin{abstract}
Automating the analysis of facial expressions of individuals is one of the challenging tasks in opinion mining. In this work, the proposed technique for identifying the face of an individual and the emotions, if present from a live camera. Expression detection is one of the sub-areas of computer visions which is capable of finding a person from a digital image and identify the facial expression which are the key factors of nonverbal communication. Complexity involves mainly in two cases viz., 1)if more than one emotions coexist on a face. 2) expressing same emotion between individuals is not exactly same. Our aim was to make the processes automatic by identify the expressions of people in a live video. In this system OpenCV library containing face recognizer module for detecting the face and for training the model. It was able to identify the seven different expressions with 75-85\% accuracy. The expressions identified are happy, sadness, disgust, fear, anger, surprise and neutral. The this an image frame from is captured from the video, locate the face in it and then test it against the training data for predicting the emotion and update the result. This process is continued till the video input exists. On top of this the data set for training should be in such a way that, it prediction should be independent of age, gender, skin color orientation of the human face in the video and also the lamination around the subject of reference.
\end{abstract}

Keywords: Computer vision, Facial emotion recognition, Human emotions, Automatic identification, HAAR classifier, Face detection, Live detection.

\section{I.INTRODUCTION}

The opinions of an individual are expressed through their facial expressions more quickly than the verbal communication. The need is these expressions are required to be analyzed at the same rate effectively to draw correct inferences so that the essentials can be fulfilled precisely thereby shrinking the gap between issues and solutions. Different reactions on face are created by the movement of muscles in different way. Presence or absence of eye contact during communication leads to different reasoning. In this work six emotions are considered viz., Happy, sad, anger, fear, disgust and surprise. Neutral is considered as the state where no emotion exits. Smile on the face is considered as happy emotion in which the eyes and muscles under them are curved upwards in shape. Sadness is identified by frowning in which the muscles in lip area are curved downwards. Anger is identified by strained eyebrows and extended eyelids. Fear is identified by increasing scantness in eyebrows. The disgust emotion is identified by lowering of eyebrows and some wrinkles on nose.

Revised Manuscript Received on November 24, 2019.

* Correspondence Author

Keerthana B.Chigateri*, Computer Science department, N.M.A.M.I.T affiliated to Visvesvaraya Technological University, Nitte,Udupi District, Karnataka, India. Email: keerthanabc@nitte.edu.in
The surprise expression in identified by stretching and spreading of muscles around eyes and mouth. If none of the above said movements are noticed, it is identified as neutral The main objectives of this work are: 1) live human emotion collection for the purpose of multi-classification in which each output will have one of the six emotions as its label one at a time. No multiple labels are assigned at the same time. The video is converted in frames and then to images. 2) Extracting the faces from the collected images and identifying the features using FisherFace algorithm. 3)Splitting the data-set into training and testing sets 4)identifying the emotion expressed by person in real time. It is implemented in OpenCV python using FisherFace algorithm and Haar classifier. Principal Component Analysis(PCA) is used for feature extraction, HSV color space for face recognition and Eucledian distance for decision making are used.

\section{RELATED WORK}

Aayushi Sharma[1] uses FNN(Feed Forward Neural networks for face recognition, PCA and ICA for feature extraction and matlab for implementation.

Qiang Hua [2] use Locality preserving Projection on Wavelet Subband for features extraction and Artificial Neural Network for recognition.

Sourabh Hanamsheth [3] study the influence of facial landmark localization in the face recognition performance and assess the impact of extracting the HOG features from a regular grid and at multiple scales.

In Dipesh Vaya [5] face recognition is carried out by a fusion of Principal Component Analysis and Mahalanobis Distance. It uses Eigen Face method for the compression or information reduction.

I.Michael Revina [6] uses Enhanced Modified Decision Based Unsymmetric Trimmed Median Filter (EMDBUTMF) method to remove the noisy pixels from the face image and presents the Local Directional Number (LDN) pattern, Dominant Gradient Local Ternary Pattern (DGLTP) descriptor for feature extraction and Support Vector Machine (SVM) classifier for classification. The histogram features are selected from face images using the LDN and DGLTP descriptors.

Dr.PriyaGupta [9] proposes a new way of using a deep neural network(another type of deep network)for face recognition on Yaleface database.

Devendra Gondole [10] first splits the face into small regions from which Local Binary Pattern (LBP) histograms are selected and linked into a single, spatially boosted feature histogram well orderly constituting the face image. 


\section{Real Time Detection and Identification of Human Emotions through Live Streaming}

\section{METHODOLOGY}

1. Pre-processing: It involves obtaining of images using web camera, and RBG image transformation to gray scale of 8 bit gray scale BMP format with size $350 \times 350$ pixels. Additionally, the face data is slit into two sections i.e. one for training image (training data-set) and another as test image (testing data-set).

At this image processing stage, Fisherface method is applied to construct feature vector of facial image data used by system In the next step feature vector of training image is compared and matched with feature vector of test image using Euclidean distance formula:

$$
d((a, b),(c, d))=\sqrt{ }(a-a)^{2}+(c-d)^{2}
$$

The dataset of the proposed project contains about 450 images collected and converted to gray scale. Sampling is arbitrary . $80 \%$ of the data is used for training and $20 \%$ is used for testing. The process is iterated for three cycles. Figure 1 shows the block diagram of the proposed system.

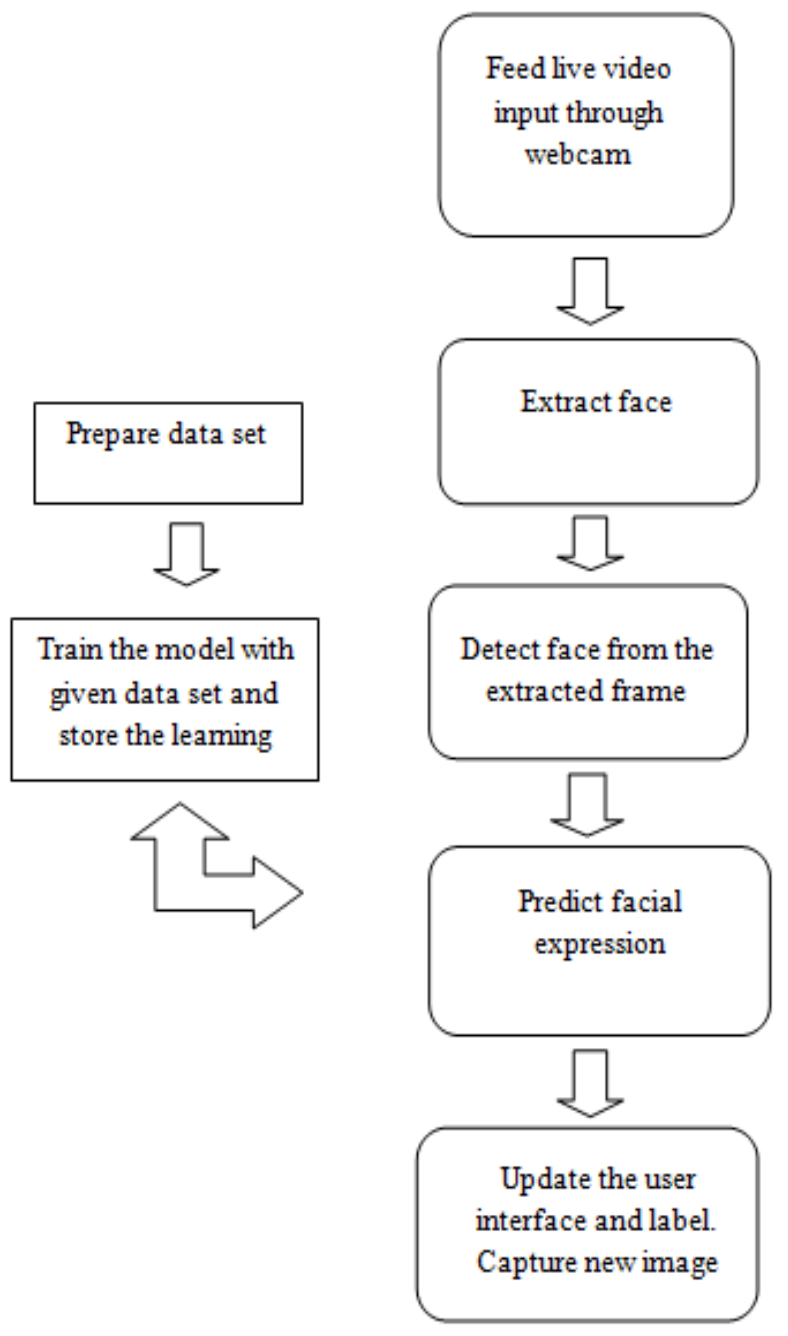

Figure 1:Block diagram of the proposed system

\section{IMPLEMENTAION}

Organizing the dataset: The Organizing module is basically the module in which the images collected from the Cohn-Kanade dataset and other face images are added into the dataset are organized into various folders depending on the emotion that is shown in those images. The CohnKanade dataset contains images of different faces that portray various emotions such as happy, sadness, fear, disgust, surprise and neutral. Figure 2 shows the organizing of datasets

The files in each of the session of each of the participant are opened. The emotions are read from those source files and then are sorted into their respective sorted set folders.

Figure 2:Block diagram for Organizing the data-sets

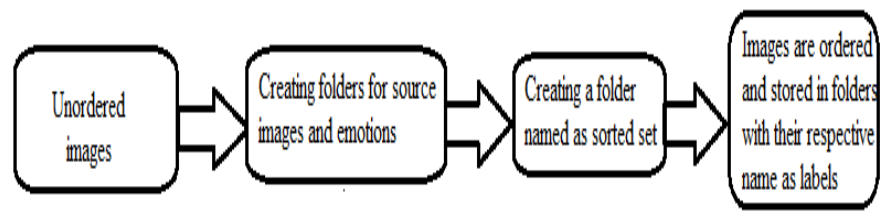

Extracting the faces: It is shown by Figure 3. the steps involved here. Extracting module in the project deals with the extraction of human face from the image in the given dataset. The faces are extracted from those images and they are converted into grey-scale. The face is cropped from those images to a resolution of $350 \times 350$ pixels. Here, compared to other existing systems, four Haar-cascade classifiers are used so as to improve the accuracy. They are given below. haarcascade_frontalface_default.xml,

haarcascade_frontalface_alt $2 . x m l$

haarcascade_frontalface_alt.xml

haarcascade_frontalface_alt_tree.xml and they are XML files that contain lines of code.

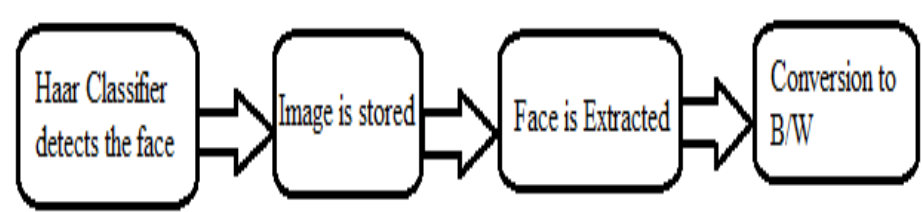

Figure 3:Block diagram for Extracting the faces

Define the emotions

def detect_faces(emotion):

Get list of all images with emotion and store it in files for $\mathrm{f}$ in files:

Open an image

Convert the image to grayscale

Detect the face using the 4 different classifiers

Go over detected faces, stop at first detected face,

return empty if no face

Crop and save the face

Get the coordinates of the rectangle containing the -face

Resize the image to $350 * 350$ pixels

for emotion in defined emotions:

detect_faces(emotion)

Training the classifier: Figure 4 shows the same. The training module in the project trains the classifier using the dataset. The dataset is divided into training set and predicting set. $80 \%$ is given for training whereas $20 \%$ is given for predicting the emotions. In this module, we use the FisherFace algorithm to extract the features of the faces and train the dataset accordingly. 


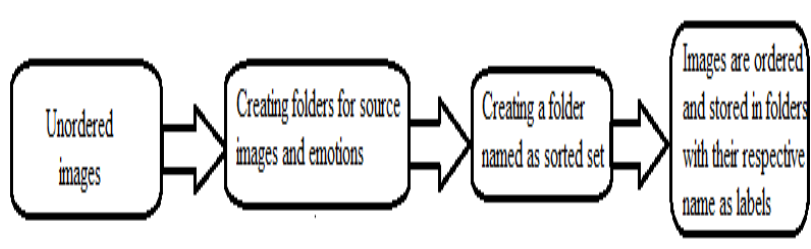

Figure 4: Block diagram for Training the classifier FisherFace method: It is enhancement of the EigenFace technique. It uses Fisher's Linear Discriminant Analysis (FLDA or LDA) for the aspect reduction. It is very handy when facial images have high variations in illumination and facial expression. The FisherFace method contains small database and the face to classify must be in the database. It utilizes the apparent features of face like mouth, nose, eye, cheekbones, chin, lips, forehead, and ears.

Initialize fisher face classifier using

FisherFaceRecognizer_create() function

def get_files(emotion):

files $=$ Define list of files

Shuffle the files

training = get first $80 \%$ of file list

prediction $=$ get last $20 \%$ of file list

return training, prediction

def run_recognizer():

Invoke the make_sets() function

fishface.train(training_data,

np.asarray(training_labels ))

fishface.save("model.xml") \#Save the trained images as image arrays in model.xml file

Calculate the correct and incorrect percentage and finally the accuracy of the project Display the accuracy

Finally, we find the accuracy also in this module by calculating the percentage of correct emotions identified divided by the total number of correct and incorrect emotions identified. The correct and incorrect emotions are based on the prediction set.1

Live Testing: This module is the final module of the project where user interaction is required. Figure 5 shows the same. The module starts with the opening of the web camera where the live streaming would take place. Each frame of that video is captured and the face is detected and extracted from that captured frame. A rectangle frame is shown around the face indicating that the face is captured and this is done using the FisherFace algorithm. The model.xml file from the training module is read in this module.

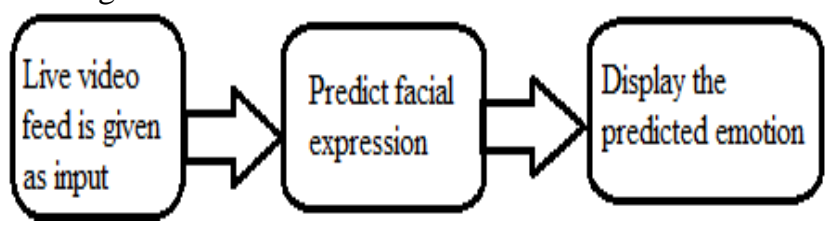

Figure 5:Block diagram for Live test

To identify the correct emotion, the video streaming should have proper lighting and should not have any obstacles covering the face to have good results. The image is then compared with the sorted data-set and the emotion is identified

Open the web camera
Start the video capturing

def livetest():

Capture the image

Convert to grayscale

Resize the image to $350 \times 350$ pixels

Predict the emotion

Update the label on the video streaming window screen The emotion can be displayed as a label on the window of the live stream. The label would keep flickering in the beginning but after sometime it will be steady and the correct emotion will be displayed.

\section{V.RESULTS}

The results mentioned below dictates how the proposed system detects each of these facial expressions. Table 1 shows the various test cases for the proposed system.

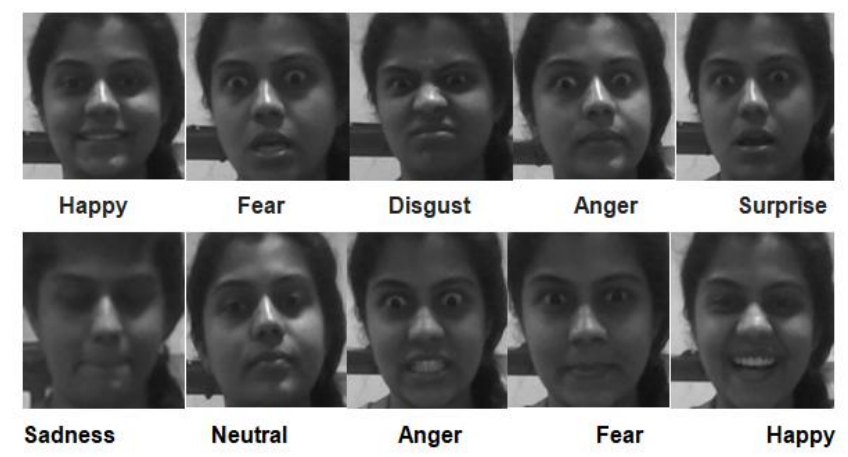

Figure 6:Samples of the outputs obtained from the facial emotion recognition system

\begin{tabular}{|c|c|c|c|c|c|}
\hline No & Test Case & Input & $\begin{array}{l}\text { Actual } \\
\text { Output } \\
\text { (X) }\end{array}$ & $\begin{array}{l}\text { Expected } \\
\text { Output } \\
(\mathrm{Y})\end{array}$ & $\begin{array}{l}X= \\
Y\end{array}$ \\
\hline 1 & $\begin{array}{l}\text { Process } \\
\text { without a } \\
\text { webcam }\end{array}$ & Nothing & Nothing & $\begin{array}{l}\text { Error } \\
\text { message }\end{array}$ & Yes \\
\hline 2 & $\begin{array}{l}\text { Giving } \\
\text { video input } \\
\text { through } \\
\text { web } \\
\text { camera }\end{array}$ & $\begin{array}{l}\text { Image } \\
\text { frame }\end{array}$ & $\begin{array}{l}\text { Detecting } \\
\text { face and } \\
\text { the } \\
\text { emotion }\end{array}$ & $\begin{array}{l}\text { Detecting } \\
\text { the } \\
\text { emotion }\end{array}$ & Yes \\
\hline 3 & $\begin{array}{l}\text { Giving } \\
\text { expressions } \\
\text { not in the } \\
\text { dataset as } \\
\text { input }\end{array}$ & Face & Neutral & Neutral & Yes \\
\hline 4 & $\begin{array}{l}\text { If no face } \\
\text { detected }\end{array}$ & Nothing & $\begin{array}{l}\text { Retain } \\
\text { the } \\
\text { previous } \\
\text { emotion }\end{array}$ & $\begin{array}{l}\text { Retain } \\
\text { the } \\
\text { previous } \\
\text { emotion }\end{array}$ & Yes \\
\hline 5 & $\begin{array}{l}\text { If face is } \\
\text { upside } \\
\text { down }\end{array}$ & Face & $\begin{array}{l}\text { Neutral } \\
\text { and } \\
\text { detects } \\
\text { face }\end{array}$ & $\begin{array}{l}\text { Detects } \\
\text { face }\end{array}$ & Yes \\
\hline
\end{tabular}

Table I: Various test cases for the proposed system 


\section{Real Time Detection and Identification of Human Emotions through Live Streaming}

\section{CONCLUSION AND FUTURE WORK}

Emotion recognition is a very important topic. There are a number of applications for this technology. The project which we have implemented here also has applications in various fields some of the applications identified are mentioned below: Security: Emotion recognition technologies can advance researchers' ability to get deep insights into consumer behavior, but it seems that they could also be applied to the security field for the purpose of recognizing an intent, for example to help recognize a terrorist waiting to act. Making cars safer and personalized: The emotion detection application can be used for detecting and alerting the drivers when they are sleepy this can prevent many of the road accidents. Smart cars can detect facial emotions and alert the driver if he looks sleepy or drowsy. Imagine your car telling you to stop for a coffee break.

Facial Emotion Detection in Interviews: All recruiters would appreciate technology that can tell them what a candidate is feeling. It can make the process of recruitment easier, also candidates who are suitable for the job both physically and mentally can be identified.

Testing Video Games: A Video game hooks you and gets your adrenaline pumping. Facial expressions while playing is a great metric to understand if the game is successful in making your experience enjoyable. Health and Hospitals: Analysis of emotions for humans can be used in patient monitoring, human-machine interaction and marketing. Some examples are:

- Old age health monitoring remotely

- Counseling

- In autism, struggling to interpret expressions

- In the case of e-learning, study the emotions and adjust the learning technique and presentation according to the style of learner

- Determining fatigue in the case of driving and alerting in advance.

Robotics: Each of the emotions expressed by human beings can be analyzed, studied and can be used to train robots. In such a manner robots will be able to express human emotions. There are developments seen in this field. Robots are being developed such that they can detect human emotions and based on the emotion expressed by the human it will play the corresponding playlist from the music players.

\section{ACKNOWLEDGMENT}

The contributes and effort of Glenn Thomas Alex, Gopa Nithish Krishna, Hariprasad Gowda, Karthika G, Kevin Cherian Kavalackal are deeply appreciated.

\section{REFERENCES}

1. Aayushi Sharma, A Hybrid Feature Extraction Technique for Face Recognition, International Journal of Advanced Research inComputer Science and Software Engineering, Volume 7, Issue 5,May2017

2. Qiang Hua, Face Recognition using Locality Preserving Projection on Wavelet Subband and Artificial Neural Network, IJCTT Volume55Number 1 January 2018

3. Sourabh Hanamsheth, Face Recognition using Histogram of Oriented Gradients, International Journal of Advance Research inComputer Science and Management Studies, January2018
4. Shraddha Arya, Face Recognitionwith Partial Face Recognition and Convolutional Neural Network, IJARCET Volume 7, Issue 1, January 2018,

5. Dipesh Vaya ,PCA BASED EFFICIENT FACE RECOGNITION TECHNIQUE, International Journal For Technological Research In Engineering, February -2018

6. I.Michael Revina,"Face expression recognition using LDN and Dominant Gradient Local Ternary Pattern descriptors", Journal of King Saud University - Computer and Information Sciences, Available online 6 April 2018

7. Devang Pandya, "COMPARATIVE ANALYSIS OF FACIAL EXPRESSION RECOGNITION USING HMM AND SVM ",International Journal of Computer Engineering \& Technology (IJCET), Volume 9, Issue 6, November-December 2018, pp. 167-180

8. Varsha D. Patil, A REVIEW ON FACE RECOGNITION TECHNIQUES, NCETET, 2018

9. Dr.PriyaGupta, Deep Neural Network for Human Face Recognition, I.J.Engineering and Manufacturing,2018

10. Devendra Gondole, Face Recognition Based On LocalBinary Pattern, 2018

11. "FACIAL EXPRESSION RECOGNITION USING FACIAL LANDMARKS" M. I. N. P. Munasinghe 2018 IEEE/ACIS 17th International Conference on Computer and Information Science (ICIS)

\section{AUTHORS PROFILE}

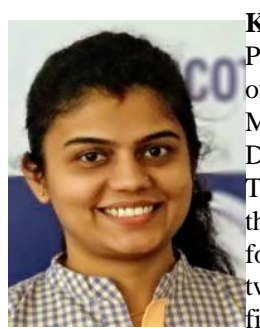

Keerthana B.Chigateri isworking as Assistant Professor in the Department of CSE, NMAM Institute of Technology, Nitte. She has completed her B.E and M.Tech in Computer Science and Engineering and Digital Electronics respectively from Vivesvaraya Technological University. She has eight years and three months of teaching experience and two years and four months of research experience. She has presented wo papers in two international conferences. Her major Intelligence. 